\title{
Clinical profiling and use of loop-mediated isothermal amplification assay for rapid detection of Mycobacterium tuberculosis from sputum
}

\author{
Poudel A ${ }^{1}$, Pandey BD ${ }^{2}$, Lekhak B ${ }^{3}$, Rijal B ${ }^{4}$, Sapkota BR ${ }^{5}$, Suzuki ${ }^{6}$
}

${ }^{1}$ Assistant Lecturer, Department of Microbiology, Kathmandu Medical College, Sinamangal, Nepal, ${ }^{2}$ Senior Medical Officer, Sukraraj Tropical and Infectious Disease Hospital, Kathmandu, Nepal, ${ }^{3}$ Assistant Professor, Central Department of Microbiology, Tribhuban University, Kathmandu, Nepal, ${ }^{4}$ Associate Professor, Department of Microbiology, Tribhuban University Teaching Hospital, Kathmandu, Nepal, ${ }^{5}$ Research Scientist, Mycobacterial Research Laboratory, Anandaban Hospital, Kathmandu, Nepal, ${ }^{6}$ Professor, Research Center for Zoonosis Control, Hokkaido University, Hokkaido, Japan.

\begin{abstract}
Background: Tuberculosis is a global health problem and the situation is worsening with newer incidences of drug resistance and HIV association. Diagnosis of tuberculosis can be done by many methods and test, culture of sputum being the ideal one. Nucleic acid amplification (NAA) assay are more time efficient one, that amplify and detect specific nucleic acid sequences allows rapid, sensitive and specific detection of $\mathrm{M}$. tuberculosis in sputum samples.

Objectives: The present study intends to compile the clinical presentations of the pulmonary tuberculosis (PTB) patients and to evaluate the efficacy of in-house loop-mediated isothermal amplification (LAMP) in detecting Mycobacterium tuberculosis in sputum samples by comparing with microscopy and culture.

Materials and methods: Two hundred two sputum samples were collected from 202 patients at National Tuberculosis Center, Bhaktapur, Nepal. Complete clinical profiling, epidemiological data and record on BCG vaccination were noted and the samples were subjected for microscopy, culture and in-house LAMP with six primers specific for 16S RNA gene of Mycobacterium tuberculosis.

Result: Of the 176 cases of clinical profiling, productive cough was most common symptom in 147 (83.52\%), followed by chest pain 136 (77.27\%), fever 133 (75.56\%) and haemoptysis 61 (34.66\%). There was a statistically significant association between BCG vaccination and development of TB $\left(\chi^{2}=5.33, \mathrm{P}=0.02\right)$. Of 202 cases, $115(56.93 \%)$ were chest X-ray positive, 101(50\%) were direct smear-positive and $100(49.51 \%)$ were culture positive. LAMP had a sensitivity of $97 \%$ and specificity of $94.12 \%$ while comparing with culture. In addition, its sensitivity and specificity were $91.09 \%$ and $89.11 \%$ respectively with reference to microscopy.

Conclusion: As in our previous study, overall, the result of present study further confirms that the in-house LAMP is a simple, rapid, sensitive and specific DNA amplification technique for PTB diagnosis. Because of rapidity of microscopy and specificity of culture, in-house LAMP assay can be used as a very powerful and useful supplementary tool with complete clinical profiling of the patients for rapid diagnosis of TB in both AFB-positive and negative cases who are suspected as PTB in disease endemic country like Nepal.
\end{abstract}

Key words: clinical profiling, Sputum, DNA, LAMP, M. tuberculosis, Nepal

$\mathrm{T}$ uberculosis (TB) is an airborne infectious disease caused by Mycobacterium tuberculosis and still remains as a major global public health problem. An estimated 1.5 million people died from TB in 2006 and another 200,000 people with HIV died from HIVassociated $\mathrm{TB}^{1}$. The situation is further exacerbated with the increasing incidence of multidrug-resistant TB (MDR-TB) and extensively drug-resistant TB (XDRTB), HIV-associated TB, and weak health delivery systems in the disease burden countries. Among the countries substantially affected by TB, Nepal has an elevated annual risk of infection, with an estimated
$45 \%$ of the total population being infected. Each year, 40,000 people develop active TB, of whom 20,000 have infectious pulmonary disease, and over 6000 people die of $\mathrm{TB}^{2}$.

\footnotetext{
Correspondence

Mr. Ajay Poudel

Assis Lecturer, Dept of Microbiology,

Kathmandu Medical College Teaching Hospital (KMCTH),

Duwakot, Bhaktapur, Nepal.

E-mail: ajy_18@hotmail.com
} 
Diagnostic process of PTB initiates with a high clinical suspicion on persistent cough, weight loss, and night sweats, which is further supported by the use of various diagnostic tools. However, clinical manifestations of PTB are non-specific and they don't help to distinguish PTB from other chest disease. Although, radiological examination of chest is usually the first diagnostic study undertaken after history and physical examination, the low specificity of chest X-rays risks high levels of over diagnosis ${ }^{3}$. Other diagnostic tests for TB include the tuberculin skin test, and sputum microscopy and cultures. If the disease is detected early and fully treated, people with the disease quickly become noninfectious and eventually cured by taking antibiotics daily for several months. The laboratory diagnosis of TB currently depends on acid-fast staining and culture of processed sputum samples, which are technologies that have been used for decades. Microscopic examination of acid-fast smears although is a rapid technique, has a sensitivity and specificity low enough to be useful only as a presumptive screening test. Culture is the gold standard test, but it is labor-intensive and slow ${ }^{4}$. In such condition, nucleic acid amplification (NAA) assay that amplify and detect specific nucleic acid sequences allows rapid, sensitive and specific detection of $M$. tuberculosis in sputum samples ${ }^{5}$.

Among several NAA assays, Loop Mediated Isothermal Amplification (LAMP) assay is a novel nucleic acid amplification method in which reagents react under isothermal condition (in the region of $64^{\circ} \mathrm{C}$ ) with high specificity, efficiency and rapidity ${ }^{6}$. This method is characterised by its use of six different primers specially designed to recognize eight distinct regions on target gene with a strand displacement reaction. It provides high amplification efficiency with DNA being amplified by $10^{9}-10^{10}$ times in $15-60$ minutes. The presence of the target gene sequence can easily be detected just by visual inspection of fluorescence or turbidity ${ }^{6,7}$. Thus, LAMP is a promising platform for the development of a simple and sensitive tool for the molecular detection of TB in developing countries and based on this, we developed an in-house LAMP method and evaluated in the resource constraints setting in $\mathrm{Nepal}^{8}$. In the present study, we used the standardised in-house LAMP method for rapid detection of M. tuberculosis in sputum samples and also evaluated the sensitivity, specificity and applicability of this method in comparison with chest X-ray, microscopy and culture. Furthermore, previous studies have not taken into consideration about clinical profile of the participants and other socio-cultural factors associated with disease outcome including risky behaviours as the predictive model of PTB. In the current study, we examined all these questions and further evaluated the efficacy and applicability of LAMP assay.

\section{Materials and methods}

\section{Study population and specimen collection}

A total of 202 respiratory samples; 101 were AFBpositive sputum samples from pulmonary TB patients and 101 AFB-negative sputum samples from patients with chest problem, cough and fever, were collected from National Tuberculosis Center (NTC), Nepal. During sampling and data collection, study objectives and expected outcomes were explained to each participant before seeking their consent. About four millilitres (ml) of mucoid or muco-purulent early morning sputum sample was collected and immediately proceed for microscopy, culture and LAMP.

\section{Microscopy and acid-fast staining}

Sputum sample was smeared directly on a slide and a standard fluorochrome method was employed for the detection of AFB. Results were interpreted based on WHO guidelines ${ }^{9}$.

\section{Concentration and culture}

The remaining sample was concentrated with Modified Petroff's method and used for culture growth and LAMP. The sample was taken in a graduated centrifuge tube and an equal volume of $4.0 \%$ Sodium hydro-oxide $(\mathrm{NaOH})$ was added and mixed well. This was incubated at $37^{\circ} \mathrm{C}$ for 10 minutes with frequent gentle mixing and centrifuged at $2500 \mathrm{xg}$ for 30 minutes. The supernatant was discarded and the remaining material was used for culture and DNA extraction. For culture, $0.1 \mathrm{ml}$ of concentrated sputum was inoculated into each of two culture tubes containing 2\% Ogawa medium. The tubes were examined for growth twice weekly for the first two weeks and once weekly thereafter up to eight weeks, after which a definitive result was obtained. If any colonies were seen at any stage, acid-fastness of bacilli was determined by smear examination of the growth. When no colonies were observed after weekly observation for eight weeks, then the result was considered as negative ${ }^{10}$.

\section{Pre-treatment and DNA extraction}

An equal volume of $2.0 \% \mathrm{NaOH}$ and $0.5 \%$ of $\mathrm{N}$-acetylL-cysteine (NALC) was added to the concentrated sputum sample and vortexed. After being left for 20 minutes to thinning out, the entire specimen was centrifuged at $10,000 \mathrm{xg}$ for 15 minutes. After discarding the supernatant, $50 \mathrm{mM}$ Tris $\mathrm{HCl}(\mathrm{pH} \mathrm{8.3)}$ was added in an amount equivalent to the original volume of thinned sputum. The pellet was re-suspended and centrifuged as before. The pellet was re-suspended in $50 \mathrm{mM}$ Tris $\mathrm{HCl}(\mathrm{pH} 8.3)$ equivalent to not less than $1 / 20$ th of the original thinned sputum. This was heat inactivated at $80^{\circ} \mathrm{C}$ for 20 minutes in a dry heat block. After cooling to room temperature, an equal volume of chloroform was 
added and mixed by vortexing and the aqueous phase was harvested and stored in the refrigerator until DNA extraction. The DNA was extracted by freeze and boil method $^{11}$. Briefly, 50ul of the above proceed sample was taken and subjected to a series of heat and cold shocks, alternately boiling for one minute at $100^{\circ} \mathrm{C}$ in dry heating block and freezing in liquid nitrogen $\left(-196^{\circ} \mathrm{C}\right)$ for one minute, for a total of 5 cycles. The extracted DNA was stored in the refrigerator for LAMP assay.

\section{Chest X-ray:}

Chest X-ray (CXR) was done for each participant and the observation of lung cavity in chest radiography was considered as X-ray positive.

\section{LAMP assay using sputum samples}

The details of the in-house loop-mediated isothermal amplification (LAMP) assay for detection of M. tuberculosis have been already described in the previous paper $^{8}$. Briefly, a set of primer comprises: outer primers F3 (CTGGCTCAGGACGAACG), B3 (GCTCATCCCACACCGC), inner primers FIP (CACCCACGTGTTACTCATGCCAGTCGAACGGAAAGGTCT) and BIP (TCGGGATAAGCCTGGACCACCAGACATGCATCCCGT), and loop primers FLP (GTTCGCCACTCGAGTATCTCCG) and BLP (GAAACTGGGTCTAAATACCGG) which recognized eight distinct regions of the $16 \mathrm{~S}$ rRNA gene of M. tuberculosis complex were used. The LAMP reactions were performed in a total volume of $25 \mathrm{ul}$ consisting of $30 \mathrm{pmol}$ each of inner primers FIP and BIP, 5 pmol each of outer primers F3 and B3, 20 pmol each of loop primers FLP and BLP, $1.4 \mathrm{mM}$ deoxynucleoside triphosphate, $0.8 \mathrm{M}$ betaine, $20 \mathrm{mM}$ Tris/ $\mathrm{HCl}$ (pH 8.8), $10 \mathrm{mM} \mathrm{KCl}, 10 \mathrm{mM}$ (NH4)2SO4, 8 mM MgSO4 and $8 \mathrm{U}$ Bst DNA polymerase (New England Biolabs) with specified amounts of bacterial DNA. The mixture was incubated at $64^{\circ} \mathrm{C}$ for an hour in a thermocycler (Minicycler ${ }^{\mathrm{TM}} \mathrm{MJ}$ Research). The result was considered positive when mixture turned into green fluorescence and it was considered as negative while it remained orange.

\section{Result}

Of the 202 participants, only 176 were available for interviewed and pre-tested structured questionnaire were used for gathering the information related to clinical and epidemiological characteristics of study participants. Out of 176 cases, $42(23.86 \%)$ cases had previous history of TB in the family and 147 (83.52\%) reported a productive cough with or without sputum. Moreover, chest pain was present in 136 (77.27\%), followed by fever $133(75.56 \%)$ and haemoptysis $61(34.66 \%)$. BCG vaccination scar suggesting previous immunization was found in $79(44.89 \%)$ and statistical analysis revealed that there was a statistically significant association between $\mathrm{BCG}$ vaccination and development of $\mathrm{TB}\left(\chi^{2}=5.33, \mathrm{P}=0.02\right)$. A numbers of predisposing factors alone or in combination were found to be associated with the disease outcome. These factors were smoking and alcohol consumption which were found in $101(57.38 \%)$ and $105(59.66 \%)$ cases respectively. Only $69(39.20 \%)$ had the knowledge on transmission of TB (Table 1).

Out of 202 patients, 115 cases $(56.93 \%)$ were positive by radiological examination (chest x-ray) and 101(50\%) were by microscopy. The culture on Ogawa medium was positive in 100 cases (49.51\%) and LAMP assay was positive in 103 cases $(50.99 \%$ ) (Table 2).

A. Comparison of LAMP with reference to microscopy: In smear positive 101 cases, LAMP was positive in 91 $\mathrm{C}^{+} \mathrm{X}^{+} ; 1 \mathrm{C}^{-} \mathrm{X}^{-}$cases. No positive LAMP was found in 2 $\mathrm{C}^{+} \mathrm{X}^{+} ; 1 \mathrm{C}^{+} \mathrm{X}^{-} ; 3 \mathrm{C}^{-} \mathrm{X}^{+}$; and $3 \mathrm{C}^{-} \mathrm{X}^{-}$cases. Similarly out of 101 smear negative, LAMP was positive in $5 \mathrm{C}^{+} \mathrm{X}^{+} ; 1 \mathrm{C}^{+}$ $\mathrm{X}^{-}$; $3 \mathrm{C}^{-} \mathrm{X}^{-}$; and $2 \mathrm{C}^{-} \mathrm{X}^{+}$cases. The sensitivity of LAMP in comparison to microscopy was $91.1 \%$ and specificity was $89.1 \%$.( Table 4 ).

B. Comparison of LAMP with reference to culture: In culture positive cases, LAMP was positive in $91 \mathrm{M}^{+} \mathrm{X}^{+}$; $5 \mathrm{M}^{-} \mathrm{X}^{+}$; and $1 \mathrm{M}^{-} \mathrm{X}^{-}$cases. LAMP was found negative in $2 \mathrm{M}^{+} \mathrm{X}^{+}$and $1 \mathrm{M}^{+} \mathrm{X}^{-}$cases. Among culture negative, LAMP was positive in $1 \mathrm{M}^{+} \mathrm{X}^{-} ; 2 \mathrm{M}^{-} \mathrm{X}^{+}$; and $3 \mathrm{M}^{-} \mathrm{X}^{-}$. The sensitivity of LAMP in comparison to culture was $97.00 \%$ and specificity was $94.12 \%$ respectively (Table 4). 
Table 1: The relevant clinical and epidemiologic characteristics of 176 patients

\begin{tabular}{|l|l|}
\hline Clinical characteristics & N $(\%)$ \\
\hline Previous history of TB on family & $42(23.86)$ \\
\hline Cough & $147(83.52)$ \\
\hline Chest pain & $136(77.27)$ \\
\hline Fever & $133(75.56)$ \\
\hline Haemoptysis & $61(34.66)$ \\
\hline BCG Vaccinated & $79(44.89)$ \\
\hline Others & \\
\hline a) Habit of smoking & $101(57.38)$ \\
\hline b) Habit of alcohol drinking & $105(59.66)$ \\
\hline c) Knowledge on transmission of TB & $69(39.20)$ \\
\hline
\end{tabular}

Table 2: Result of 202 specimens by microscopy, culture, LAMP and X-ray

\begin{tabular}{|l|c|c|}
\hline Test & Positive N (\%) & Negative N (\%) \\
\hline Microscopy $(\mathrm{M})$ & $101(50 \%)$ & $101(50 \%)$ \\
\hline Culture $(\mathrm{C})$ & $100(49.5 \%)$ & $102(50.5 \%)$ \\
\hline LAMP & $103(50.99 \%)$ & $99(49.01 \%)$ \\
\hline X-Ray $(\mathrm{X})$ & $115(56.93 \%)$ & $86(43.07 \%)$ \\
\hline
\end{tabular}

Table 3: Comparative result of microscopy, culture. LAMP and X-ray

\begin{tabular}{|c|c|c|c|c|}
\hline Microscopy (M) & Culture (C) & LAMP & X-ray (X) & Number (\%) \\
\hline+ & + & + & + & $91(45.05)$ \\
\hline+ & + & + & - & $0(0.00)$ \\
\hline+ & + & - & - & $2(0.99)$ \\
\hline+ & + & - & + & $1(0.49)$ \\
\hline+ & - & - & - & $3(1.48)$ \\
\hline+ & - & + & + & $1(0.49)$ \\
\hline+ & - & - & + & $12(5.94)$ \\
\hline- & - & + & + & $2(0.99)$ \\
\hline- & - & + & - & $5(2.48)$ \\
\hline- & + & + & - & $3(1.48)$ \\
\hline- & - & + & - & $1(0.49)$ \\
\hline- & + & - & & $78(38.61)$ \\
\hline
\end{tabular}

Table 4: Comparison of LAMP with reference to microscopy and culture

\begin{tabular}{|l|lll|lll|}
\hline & \multicolumn{3}{|c|}{ Microscopy } & \multicolumn{2}{c|}{ Culture } \\
\hline & + & - & total & + & - & Total \\
\hline LAMP + & 92 & 11 & 103 & 97 & 6 & 103 \\
\hline LAMP- & 9 & 90 & 99 & 3 & 96 & 99 \\
\hline Total & 9 & & 100 & 102 & 202 \\
\hline Sensitivity & 101 & 101 & 202 & $97.00 \%$ & \\
\hline Specificity & $91.1 \%$ & $94.12 \%$ \\
\hline Positive predictive value & $89.1 \%$ & $94.18 \%$ \\
\hline Negative predictive value & $89.3 \%$ & $96.97 \%$ \\
\hline
\end{tabular}




\section{Discussion}

The main findings of our present study are: productive cough is the most common symptom in clinical profiling and there is a significant association between BCG vaccination and development of TB. Furthermore, family history, smoking and alcohol consumption are the other risky behaviours associated with the PTB development. The present study further demonstrated the simplicity, rapidity, sensitivity and specificity of LAMP assay for rapid diagnosis of TB in resource constraints setting of developing country like Nepal.

Based on clinical profile and the information on risky behaviours of study participants, the main underlying clinical presentation for tuberculosis was found to be a productive cough $(83.52 \%)$, followed by chest pain (77.27\%), fever (75.56\%) and haemoptysis (34.66\%). However, in a study in India, common clinical symptoms amongst tuberculosis are fever $(87.5 \%)$ as the major common symptoms, followed by cough $(68.7 \%)$, weight loss $(56.25 \%)$, pain chest $(43.7 \%)$, haemoptysis $(25 \%)^{12}$. In our study, out of $176,44.89 \%$ were immunized with BCG vaccination and statistical analysis revealed that $\mathrm{BCG}$ non-receiver were more likely to suffer with TB as compared to receiver since the values were statistically significant $\left(\chi^{2}=5.33\right.$, $\mathrm{P}=0.02)$. However, Centres for disease control and prevention, Atlanta, USA has reported the vaccination with BCG is significantly associated with a reduction in the incidence of pulmonary tuberculosis. Its two meta-analyses of the published results of BCG vaccine clinical trials and case-control studies have confirmed that the protective efficacy of $\mathrm{BCG}$ for preventing serious forms of $\mathrm{TB}$ in children is high (i.e., $>80 \%$ ). These analyses, however, cannot clarify demonstrate the protective efficacy of BCG for preventing pulmonary TB in adolescents and adults; this protective efficacy is variable and equivocal ${ }^{13}$.

In laboratory diagnosis, out of 101 smear positive cases, 91 cases were also positive by LAMP, culture and X-ray; however, one LAMP positive was found to be negative in both culture and X-ray. LAMP positivity in this case may be due to its high sensitivity. Culture negativity in this case may be due to over decontamination with $\mathrm{NaOH}$ during sample processing. Of the remaining 9 smear positive cases, three were LAMP, culture, and $\mathrm{X}$-ray negative, and three LAMP and culture negative but X-ray positive. The result here indicated that they were other than M. tuberculosis. The X-ray positive result is due to cavities usually develops because of the immune response to the tubercular bacilli leading to destruction of lung tissue. Its negative result may be due to lack of fully functioning immune system, like in HIV cases, where there is less tissue destruction and hence less lung cavitations ${ }^{14}$.
Likewise, among 101 smear negative cases, $M$. tuberculosis was detected by LAMP in five both culture and X-ray positive cases; and one culture positive but $\mathrm{X}$-ray negative case. The positive result of LAMP is in comparable with that of culture, emphasizing the specificity of LAMP. But, five LAMP positive cases were culture negative which were probably due to killing of mycobacterium present in very less number in sputum samples. Here, LAMP positive result also could be due to the ability of the LAMP test to detect very low number and even dead bacteria in a sample which can be present in a symptomatic individual. Of remaining 90 smear negative cases, all were found to be negative by both LAMP and culture. This finding highlighted the specificity of LAMP. Thus, LAMP combines the rapidity of microscopy with specificity of culture and will be helpful in early treatment and proper case management.

While comparing the LAMP with culture as gold standard, the sensitivity of LAMP was $97 \%$, specificity $94.12 \%$, predictive value of positive test $94.18 \%$, predictive value of negative test $96.97 \%$, percentage of false negative $3 \%$, and percentage of false positive value $5.88 \%$. This demonstrates a high sensitivity, specificity, and predictive values of LAMP as compared with that of culture. Percentage of false negative and percentage of false positive are very low indicating the higher accuracy of the test. Similarly, comparative analysis of LAMP with reference to microscopy revealed that the LAMP had sensitivity $91.09 \%$, specificity $89.11 \%$, predictive value of positive test $89.32 \%$, predictive value of negative test $90.91 \%$, percentage of false negative $8.91 \%$, and percentage of false positive $10.89 \%$ respectively. The higher sensitivity, specificity, of LAMP in this case indicates that LAMP is superior to the acid-fast smear test. This result is comparable with our previous study, which shows that $100 \%(96 / 96)$ sensitivity and $94.2 \%$ (98/104) specificity against culture as gold standard method $^{8}$. Similarly, in another study, investigators have established a LAMP method using six primers to be a highly sensitive and specific means of diagnosing pulmonary tuberculosis. The sensitivity study of LAMP on sputum sample using purified DNA indicated that the LAMP-assay has a detection limit equivalent to that of Amplicor test. However, in some culture positive sputum samples, their system was unable to detect $M$. tuberculosis ${ }^{6}$.

Boehme et al. evaluated a prototype LAMP assay with simplified manual DNA extraction for the detection of pulmonary TB in microscopy centres and demonstrated that the sensitivity of LAMP in smear- and culturepositive sputum specimens were $97.7 \%$ and in smearnegative, culture-positive specimens was $48.8 \%$. The specificity in culture-negative samples was $99 \%{ }^{15}$. But in our present study, the sensitivity of LAMP was 
97\% and 91.09\% while comparing with culture and microscopy respectively. This result further confirmed the high sensitivity and specificity of in house LAMP assay for rapid detection of $M$. tuberculosis sputum samples. Although this study tested small numbers of patients, the outcome informs LAMP assay to have great advantages of confirmatory diagnosis of clinically, microscopically and culturally confusing cases, thus facilitating effective treatment and case management. However, further study taking more samples can be done for improving sensitivity, specificity and reproducibility of this test and to make it more users friendly and cost effective.

\section{Conclusion}

In conclusion, keeping in view of high incidence and prevalence of tuberculosis, patients should always be screened for specific symptoms and BCG vaccination during the diagnosis of tuberculosis in disease endemic country like Nepal. The LAMP assay used in this study is a novel nucleic acid amplification method that allows direct identification of M. tuberculosis in sputum samples. Due to its easy operation with high sensitivity and specificity, it will be simple enough to use in well-equipped laboratories with adequate bio-safety arrangement if facilities such as sample preparation, nucleic acid extraction, and cross-contamination controls are addressed. It has a great potential to improve the clinicians' ability to diagnose tuberculosis.

\section{Acknowledgment}

We would like to express our acknowledgment to Ms. Sarsawoti Khadgi, Mr Chaman Ranjit, Dr. Murdo Macdonald at Mycobacterial Research Laboratory, Anandaban Hospital, and Mr. Kiran Pandey of Everest International Clinic and Research Center, Kathmandu, Nepal, for their technical assistance. We are also thankful to Mr. Dhurba Khadka and staffs of the Nepal Tuberculosis Center for providing the laboratory facilities for microscopy and culture for this study. We are very much grateful to all members of Japanese team for their important support in this study.

\section{References}

1. Tuberculosis [homepage on Internet]. World Health Organisation. Available from: http:// www.who.int/tb/en

2. National Tuberculosis Program. Annual report of National Tuberculosis Control Program Nepal. Ministry of Health, Department of Health Services. 2004.

3. World Health Organization, Stopping tuberculosis. New Delhi : WHO regional office for South-East Asia; 2002.

4. Kent BD and Kubica GP. Public health Mycobacteriology: a guide for the level III laboratory. Atlanta: U.S. Department of Health and Human Services, Centers for Disease control; 1985.

5. Centers for Disease Control. National action plan to combat multi drug-resistant tuberculosis: summary of a conference; management of persons exposed to multidrug-resistant tuberculosis. Morbid. Mortal. Weekly Rep. 1992.41:5-48.

6. Iwamoto T, Sonobe $\mathrm{T}$ and Hayashi K. Loopmediated isothermal amplification for direct detection of Mycobacterium tuberculosis complex, M. avium, and M. intracellulare in sputum samples. J. Clin. Microbiol. 2003; 41(6):2616-22.

7. Eiken Genome [homepage on the internet]. Eiken Chemical Co. Ltd. The principle of LAMP method. Available from : http:// eiken. co.jp

8. Pandey BD, Poudel A, Yoda T, Tamaru A, Oda N, Fukushima Y, et al. Development of an inhouse loop-mediated isothermal amplification (LAMP) assay for detection of Mycobacterium tuberculosis and evaluation in sputum samples of Nepalese patients. J Med Microbiol. 2008; 57: 439-43.

9. World Health Organization. Laboratory services in tuberculosis control. Part II: microscopy. Geneva, Switzerland: WHO;1998.

10. World Health Organization. Laboratory services in tuberculosis control. Part III: culture. Geneva, Switzerland: WHO;1998.

11. Sapkota BR, Ranjit C, Macdonald M. Rapid differentiation of Mycobacterium tuberculosis and Mycobacterium leprae from sputum by polymerase chain reaction. Nep Med College. J 2007; 9(1):12-6.

12. Sharma S, Singh JP, Singh R, Gandotra D, Gupta R. Clinical spectrum of pulmonary and extrapulmonary tuberculosis in end stage renal disease in Jammu. J Science. 2008; 10 (1): $11-4$.

13. Centers for Disease Control (CDC). The role of BCG vaccine in the prevention and control of tuberculosis in the United States. MMWR. 1996; 45. RR-4.

14. World Health Organization. TB: A clinical manual for South East Asia. Geneva, Switzerland: WHO; 1997.

15. Boehme CC, Nabeta P, Henostroza G, Raqib R , Rahim Z ,Gerhardt M, et al. Operational feasibility of using loop-mediated isothermal amplification for diagnosis of pulmonary tuberculosis in microscopy centers of developing countries. J Clin Microbiol. 2007, 45, 1936-40. 